\title{
Transcriptome analysis and de novo annotation of the critically endangered Amur sturgeon (Acipenser schrenckii)
}

\author{
X.J. Zhang ${ }^{1,2,3}$, H.Y. Jiang ${ }^{1,2,3}$, L.M. Li ${ }^{1,2,3}$, L.H. Yuan ${ }^{1,2,3}$ and J.P. Chen ${ }^{1,2,3}$ \\ ${ }^{1}$ Guangdong Entomological Institute, Guangzhou, China \\ ${ }^{2}$ Guangdong Public Laboratory of Wild Animal Conservation and Utilization, \\ Guangzhou, China \\ ${ }^{3}$ Guangdong Key Laboratory of Integrated Pest Management in Agriculture, \\ Guangzhou, China
}

Corresponding author: J.P. Chen

E-mail: chenjp@gdei.gd.cn

Genet. Mol. Res. 15 (2): gmr.15027999

Received November 5, 2015

Accepted January 18, 2016

Published June 21, 2016

DOI http://dx.doi.org/10.4238/gmr.15027999

\begin{abstract}
The aim of this study was to provide comprehensive insights into the genetic background of sturgeon by transcriptome study. We performed a de novo assembly of the Amur sturgeon Acipenser schrenckii transcriptome using Illumina Hiseq 2000 sequencing. A total of 148,817 non-redundant unigenes with base length of approximately $121,698,536 \mathrm{bp}$ and ranges from 201 to $26,789 \mathrm{bp}$ were obtained. All the unigenes were classified into 3368 distinct categories and 145,449 singletons by homologous transcript cluster analysis. In all, 46,865 (31.49\%) unigenes showed homologous matches with $\mathrm{Nr}$ database and 32,214 (21.65\%) unigenes were matched to Nt database. In total, 24,862 unigenes were categorized into significantly enriched 52 function groups by $\mathrm{GO}$ analysis, and 38,436 unigenes were classified into 25 groups by KOG prediction, as well as 128 enriched KEGG pathways were identified by 45,598 unigenes $(\mathrm{P}<0.05)$. Subsequently, a total of 19,860 SSRs markers were identified with the abundant di-
\end{abstract}


nucleotide type $(10,658 ; 53.67 \%)$ and the most AT/TA motif repeats $(2689 ; 13.54 \%)$. A total of 1341 conserved lncRNAs were identified by a customized pipeline. Our study provides new sequence and function information for A. schrenckii, which will be the basis for further genetic studies on sturgeon species. The huge number of potential SSRs and putatively conserved lncRNAs isolated by the transcriptome also shed light on research in many fields, including the evolution, conservation management, and biological processes in sturgeon.

Key words: Amur sturgeon; Transcriptome sequencing; Simple sequence repeat; Long non coding RNA; Function annotation

\section{INTRODUCTION}

Sturgeon are polyploid chondrostean fishes originating in the Devonian period and belong to the order Acipenseriformes, which contains 27 species divided into two families, including the Acipenseridae (sturgeon, 25 species) and the Polydontidae (paddlefish, 2 species). Sturgeon are praised as precious "living fossils" and thus play a key role in phylogenetic studies of vertebrate evolutionary history (Bemis et al., 1997). In a March 2015 press release, the International Union for Conversation of Nature and Natural Resource (IUCN) Red List identified sturgeon as one of the most endangered groups of animals with $85 \%$ of species being on the verge of extinction (http://www.iucnredlist.org/search). Meanwhile, sturgeons have globally been considered as excellent candidates for aquaculture due to their high commercial value from production of meat and caviar. Therefore, conflicting issues between the severe decline in natural populations and the huge profits of commercial sturgeon farming are the main driving force promoting the development of sturgeon aquaculture. To date, more than 17 sturgeon species have been farmed and progress in artificial propagation technology has accelerated the development of sturgeon aquaculture.

Sturgeon conservation is crucial as the species plays an important role in maintaining biological diversity and ecological stability. So far, the genomic information of all sturgeon species remains incomplete. As a key first step, comprehensive understanding of genetic backgrounds of sturgeon species is required. With developments in molecular techniques, studies on genetic diversity, population structure, and genetic variation, as well as marker assisted selective breeding based on simple sequence repeat (SSR) and single nucleotide polymorphism techniques (Hu et al., 2010; Senturk Akfirat and Uncuoglu, 2013; Zhang et al., 2013) have been successfully performed. However, the available genetic information is insufficient for unannotated regions; therefore, large numbers of novel molecular markers for the sturgeon database need to be updated, which may provide more suitable messages for the identification of selection breeding in sturgeon.

Transcriptome study not only provides the genetic background for gene structure, function annotation, and discovery of novel genes, but also determines when genes are expressed and how they are regulated (Jin et al., 2013). Compared with Tiling microarray and cDNA or EST sequencing, next-generation sequencing technologies developed by Illumina/ Solexa can generate over one billion bases of high-quality sequences per run at less than $1 \%$ of the cost of capillary-based methods, and is expected to dominate in future analysis of 
eukaryotic transcriptomes (Morozova and Marra, 2008; Ozsolak and Milos, 2011). Currently, transcriptome analysis is being widely applied to elucidate genetic markers of immune, reproduction-related, or economically significant traits and for genetic diversity management in cultured fish species (Richards et al., 2013; Vidotto et al., 2013; Che et al., 2014).

In the present study, we used Illumina Hiseq 2000 sequencing to assemble and annotate a transcriptome dataset from the critically endangered Amur sturgeon (Acipenser schrenckii), which is largely found in the Amur River basin and now is an important representative species in Chinese sturgeon aquaculture.

\section{MATERIAL AND METHODS}

\section{Preparation of samples, construction of cDNA library and sequencing}

Five major tissue types (liver, spleen, muscle, heart, and brain) were collected from a 5-month-old Amur sturgeon from the Engineering and Technology Center of Sturgeon Breeding and Cultivation, Chinese Academy of Fishery Science (Beijing, China). All the experimental animal procedures were approved by the Ethics Committee of Animal Experiments of Guangdong Entomological Institute. The sturgeon individual was anesthetized with eugenol and painlessly killed using practiced methods (Yuan et al., 2014). The total RNA were separately extracted from five tissue samples with RNAiso plus (TaKaRa, Shiga, Japan) according to the manufacturer's instructions. Total RNA degradation and contamination were monitored on 1\% agarose gels. Total RNA concentration was measured using Qubit ${ }^{\circledR}$ RNA Assay Kit in Qubit $^{\circledR}$ 2.0 Flurometer (Life Technologies, CA, USA). Total RNA integrity number (RIN) was assessed using the RNA Nano 6000 Assay Kit of the Bioanalyzer 2100 system (Agilent Technologies, CA, USA) and the criteria for the RNA samples were 2100 RIN $>$ 8.0. Qualified RNAs were used for further construction of cDNA library.

A $3 \mu \mathrm{g}$ RNA pooled sample with equal volumes of each of the above five tissues was used as input material for the RNA sample preparations. Sequencing libraries were constructed using Illumina TruSeq ${ }^{\mathrm{TM}}$ RNA Sample Preparation Kit (Illumina, San Diego, CA, USA) following manufacturer's instructions. In order to select cDNA fragments preferentially of $200 \mathrm{bp}$ in length, the library fragments were purified with AMPure XP system (Beckman Coulter, Beverly, MA, USA). DNA fragments with ligated adaptor molecules on both ends were selectively enriched using Illumina PCR Primer Cocktail in a 10-cycle PCR. Products were purified (AMPure XP system) and quantified using the Agilent high-sensitivity DNA assay on the Agilent Bioanalyzer 2100 system. The clustering of the index-coded samples was performed on a cBot Cluster Generation System using TruSeq PE Cluster Kit v3-cBotHS (Illumina) according to the manufacturer's instructions. Following cluster generation, the library preparations were sequenced on an Illumina Hiseq 2000 platform and 100-bp pairedend reads were generated. All cDNA data series were submitted to NCBI Sequence Read Archive (SRA) database (accession No. SRR1131121).

\section{Cleaning and assembly}

First, raw data in FASTQ format were processed by Perl script pipelines. After removing reads containing adapter, those containing ploy-N and low-quality reads from raw 
data, the clean data were obtained. At the same time, Q20, Q30, GC-content, and sequence duplication level of the clean data were calculated. All the downstream analyses were based on clean data of high quality.

Transcriptome assembly was accomplished based on the left.fq and right.fq using Trinity (Grabherr et al., 2011) with min_kmer_cov set to 2 and all other parameters set default. The credible transcription sequences were obtained using a chart analysis in the Butterfly software. The initial unigenes were gained by parsing the subtypes and sources of different transcriptions with the same gene transcription. Then, after removing redundancy, length splicing and homologous transcription clustering analysis for the initial unigenes yielded the final non-redundant unigenes, which were classified into two groups: clusters and singletons.

\section{Identification and analysis of SSRs}

All unigenes were analyzed by using the MISA computer program (MicroSAtellite) for identification of SSR motifs having mononucleotide to hexanucleotide repeats (Garg et al., 2011; Yang et al., 2011; Zhang et al., 2012). The parameters used for SSRs included at least 6 repeats for di- and 5 repeats for tri-, tetra-, penta-, hexa-nucleotide. Other settings were default parameters.

\section{Function annotation}

All the unigenes were referenced against $\mathrm{Nt}, \mathrm{Nr}$ and Swiss-Prot databases using BLASTx and BLASTn algorithm with an E-value cut-off of 1.0E-5. Based on Nr annotation, the Blast2GO software were used for Gene Ontology (GO) annotation (Conesa et al., 2005), and GO-enrichment analysis was performed to obtain the distribution of significant function groups. The unigenes were further aligned to the COG database (http://www.ncbi.nlm.nih. gov/COG/) and Kyoto Encyclopedia of Genes and Genomes (KEGG) pathway database for function assignment (Tatusov et al., 2003).

\section{Filtering and conservation analysis of long noncoding RNA transcripts}

Open reading frames (ORFs) of all the unigenes were predicted by the trans-Decoder package (http://transdecoder.github.io/), with the minimum ORF length of $100 \mathrm{bp}$. Then, all the redundant transcripts with ORFs $<300 \mathrm{nt}$ were retained. The transcripts mapped with Pfam RNAs were filtered and only transcripts with length $>200$ nt were retained. Then, the proteincoding capacity of the remaining transcripts were calculated using coding potential calculator (CPC), which incorporates the sequence features to assess the protein-coding potential of each transcript (Kong et al., 2007). CPC criteria are suggested to be a convincing approach for distinguishing noncoding RNAs from protein-coding (Li et al., 2012; Young et al., 2012). Therefore, the putative transcripts with a CPC score $<-1$ were retained as putative lncRNAs for further analysis. Subsequently, those transcripts mapped with known database [Nt, Nr, KEGG, and euKaryotic of Orthologous Group (KOG)] were further filtered.

The conservation analysis of candidate lncRNAs was performed using the method based on sequence similarity, which can identify a subset of putative lncRNA orthologs between mammals and fishes (Basu et al., 2013). All above retained unigenes were compared with Noncoding RNA Database v3.0 (http://www.bioinfo.org/noncode/) in order to align 
sequence similarity with known lncRNAs in other vertebrates (BLASTn E $<1.0 \mathrm{E}-5$ ), so putatively conserved lncRNAs from Amur sturgeon were identified.

\section{RESULTS}

\section{Cleaning and assembly}

Deep sequencing by Illumina Hiseq 2000 run generated a total of 58,940,154 and 200bp paired-end raw reads from $A$. schrenckii. After eliminating adapter sequences and filtering out the low-quality reads (those with fewer than 25 bp per read) and containing $\mathrm{N}$ content over $10 \%$ reads, the Illumina Hiseq 2000 sequencing yielded a total of 50,142,328 high-quality reads, which comprised $85.07 \%$ of the raw data. The average GC content calculated for whole dataset was $49.56 \%$ and the cleaned reads attained Q20 was $95.56 \%$. The main features of the reads of the Illumina Hiseq 2000 sequencing preprocessing step are summarized in Table 1. Therefore, the clean data were assembled de novo using the Trinity program resulting in a total of 148,817 unigenes with base length of $121,698,536 \mathrm{bp}$, ranging from 201 to 26,789 bp. Among these unigenes, 76,862 (51.67\%) were less than $400 \mathrm{bp}$ in length, 56,686 (38.09\%) were in the length range of 401-2000 bp, and 15,353 (10.32\%) were longer than $2001 \mathrm{bp}$ (Figure 1). The GC content of total unigenes had an average of $44.1 \%$. All non-redundant unigenes were divided into two clade categories, comprised of 3368 distinct clusters and 145,449 singletons. Detailed information on the A. schrenckii transcriptome squencing reads assembled by the Trinity program are summarized in Table 2 .

Table 1. Statistics of sequencing reads preprocessing for Acipenser schrenckii library.

\begin{tabular}{l|c}
\hline Index & Results \\
\hline Number of raw reads & $58,940,154$ \\
\hline Number of cleaned reads & $50,142,328$ \\
\hline Percentage of cleaned reads & $85.07 \%$ \\
\hline Average GC content & $49.56 \%$ \\
\hline Percentage of cleaned reads attainted Q20 & $95.56 \%$ \\
\hline
\end{tabular}

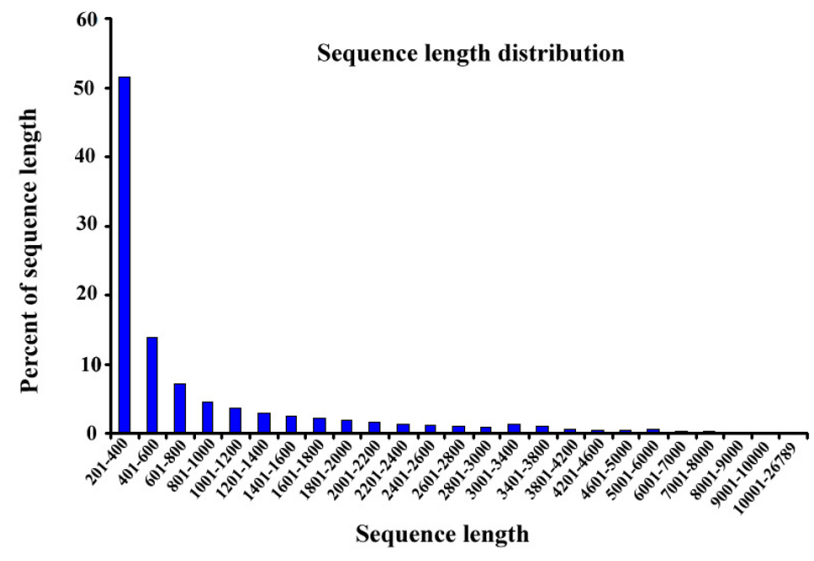

Figure 1. Length distribution of the assembled unigenes for Acipenser schrenckii. 
Table 2. Summary of Acipenser schrenckii transcriptome sequencing reads assembled de novo by the Trinity program.

\begin{tabular}{l|c}
\hline Index & Number \\
\hline Total unigenes & 148,817 \\
\hline Total base & $121,698,536$ \\
\hline Total singletons & 145,449 \\
\hline Total unigene clusters & 3368 \\
\hline N50 & 1599 \\
\hline N90 & 292 \\
\hline Maximum length & 26,789 \\
\hline Minimum length & 201 \\
\hline GC content (\%) & 44.1 \\
\hline
\end{tabular}

\section{Identification of molecular markers (SSRs)}

Related research shows that transcriptome SSR markers exhibit high-interspecific transferability (Wei et al., 2011; Che et al., 2014). Using the MISA Perl script, a total of 17,789 non-redundant unigenes (11.95\%) containing 19,860 SSRs were identified from 148,817 unigenes of sturgeon transcriptome, with 3434 of the sequences containing more than one SSR. The 17,789 unigenes were composed of 17,083 singletons (96.03\%) and 706 distinct clusters (0.97\%). Among 19,860 SSRs, the most abundant type of the repeat motif were di-nucleotide repeats $(10,658 ; 53.67 \%)$, followed by tri- $(7706 ; 38.80 \%)$, tetra- $(1460 ; 7.35 \%)$, penta- $(21$; $0.11 \%)$, and hexa-nucleotide $(15 ; 0.08 \%)$ repeat units. The length distribution of SSRs based on the number of repeat units are summarized in Table 3. The most common SSR motif types among the di-nucleotide repeats were TA/AT $(2689 ; 13.54 \%)$, followed by GT/TG $(2564 ; 12.91 \%)$, AC/ CA $(2047 ; 10.31 \%)$, and CA/AG (1904; 9.59\%). The most common SSR motif types among the tri-nucleotide repeats were ATA/TAA/AAT $(1562 ; 7.87 \%)$, followed by TAT/ATT/TTA (1452; $7.31 \%)$ and GGA/CAG/AGC (1025; 5.16\%). All the other motif types were uncommon (2321) comprising $11.69 \%$ of the total. The detailed results of SSR motif types are exhibited in Figure 2.

Table 3. Length distribution of SSRs based on the number of repeat units.

\begin{tabular}{|c|c|c|c|c|c|c|}
\hline \multirow[t]{2}{*}{ Repeat number } & \multicolumn{5}{|c|}{ Motif length } & \multirow[t]{2}{*}{ Total } \\
\hline & Di- & Tri- & Tetra- & Penta- & Hexa- & \\
\hline 5 & & 3249 & 1367 & 10 & 2 & 4628 \\
\hline 6 & 3469 & 2935 & 91 & 2 & 6 & 6503 \\
\hline 7 & 2176 & 1487 & 1 & 2 & 1 & 3667 \\
\hline 8 & 1730 & 34 & & 2 & 1 & 1767 \\
\hline 9 & 1699 & & & 1 & 5 & 1705 \\
\hline 10 & 1313 & & & 1 & & 1314 \\
\hline 11 & 258 & & & 1 & & 259 \\
\hline 12 & 13 & & & 2 & & 15 \\
\hline 13 & & & 1 & & & 1 \\
\hline$>14$ & & 1 & & & & 1 \\
\hline Total & 10,658 & 7706 & 1460 & 21 & 15 & 19,860 \\
\hline
\end{tabular}

\section{Function annotation analysis by GO, COG and KEGG}

All unigenes were compared with non-redundant nucleotide sequences and protein databases in NCBI using BLASTx and BLASTp at an E-value $<10^{-5}$. A total of 46,865 nonredundant transcripts of 148,817 unigenes, which matched the $\mathrm{Nr}$ database, were annotated. The function of all 46,865 annotated unigenes were further analyzed using GO, KOG, and KEGG. 


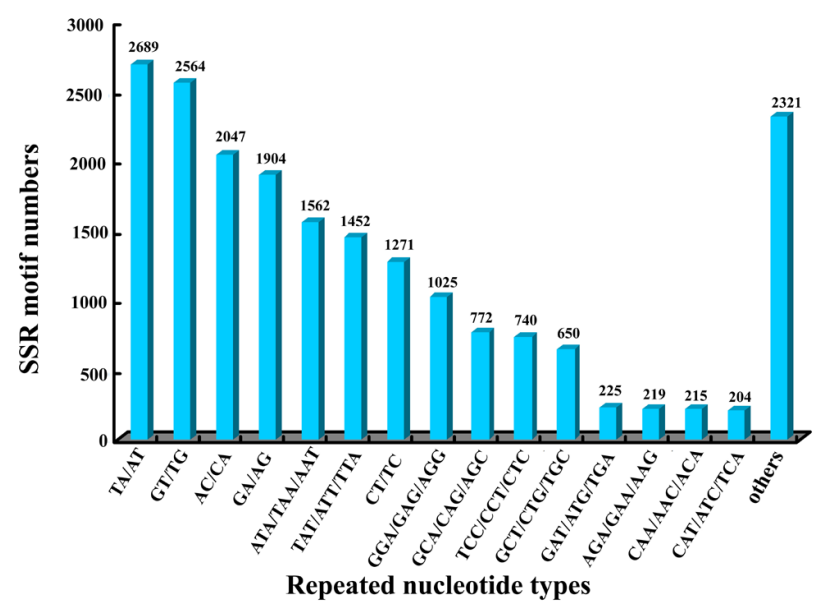

Figure 2. Type distribution of SSRs motif for Acipenser schrenckii.

The GO system provides gene function classification and research background, and divides GO terms into three categories, including biological process, cellular component, and molecular function. In the present study, a total of 24,862 unigenes were assigned to GO terms based on BLAST matches to proteins with known functions. Among the non-redundants, 20,631 sequences were assigned to 7899 terms in biological process, 20,195 sequences to 1060 terms in cellular component, and 20,338 sequences to 2833 terms in molecular function. Many unigenes were assigned to more than one GO term. For example, in the biological process category, the number of GO terms ranged from 1 to 132 for each unigene, from 1 to 26 in the cellular component category, and from 1 to 30 in molecular function. The distribution relationships of all annotated unigenes were analyzed to determine enriched GO terms by calculating super-geometry in GO categories. The results showed that 52 function groups were significantly enriched, including 20 molecular functions, 12 biological process, and 20 cellular component groups (Figure 3).

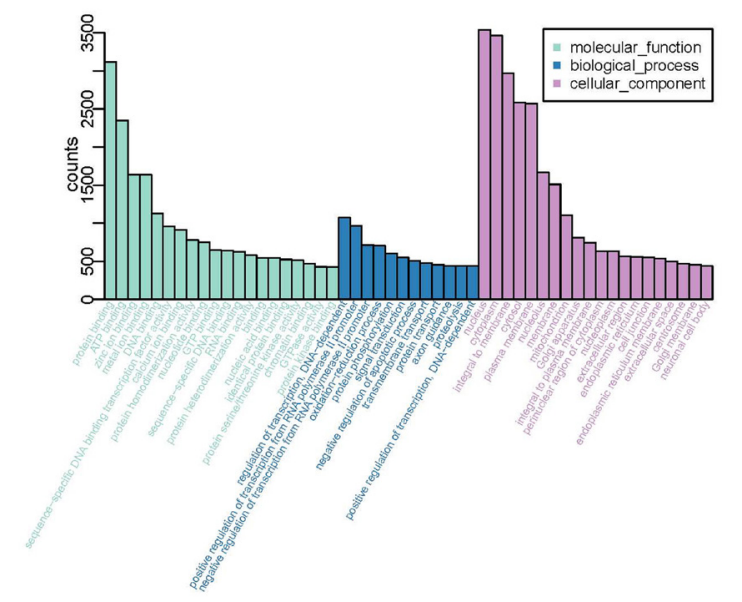

Figure 3. Top enriched GO categories annotated for assembled unigenes in Acipenser schrenckii. A total of $52 \mathrm{GO}$ terms were displayed, including 20 molecular function groups, 12 biological process, and 20 cellular component. 
In addition, we compared all unigenes with the KOG eukaryote database and found a total of 38,436 unigenes mapped into 49,363 known proteins in the database. General knowledge of all the mapped unigenes from analyzing function distribution characteristics were understood in A. schrenckii. The results showed that these mapped unigenes were classified into 25 functional categories based on their predicted functions. Among the 25 functional categories, a cluster for general function prediction only represents the largest group, followed by signal transduction mechanisms and cytoskeleton group. The clusters for nuclear structure, cell motility, and coenzyme transport and metabolism represent the smaller groups (Figure 4). Similar to the data in the GO categories, the total number of KOG sequences was $464,799(>38,436)$, because each of the unigenes were involved in more than one of the KOG functional categories.

To better understand complex networks in regulation function, an enrichment analysis of KEGG pathway was performed. A total of 45,598 unigenes were matched to the KEGG pathway database and mapped onto 289 pathway groups. Among these, the 128 pathway groups from a total of 7737 unigenes were significantly enriched $(\mathrm{P}<0.05)$ and were further summarized into six major classes: Human Diseases $(2363 ; 30.54 \%)$, Organismal System (2248; 29.06\%), Environmental Information Processing (1185; 15.32\%), Cellular Processes $(1050 ; 13.57 \%)$, Genetic Information Processing (712; 9.20\%), and Metabolism (179; 2.31\%). Detailed information on enriched KEGG pathway are given in Figure 5.

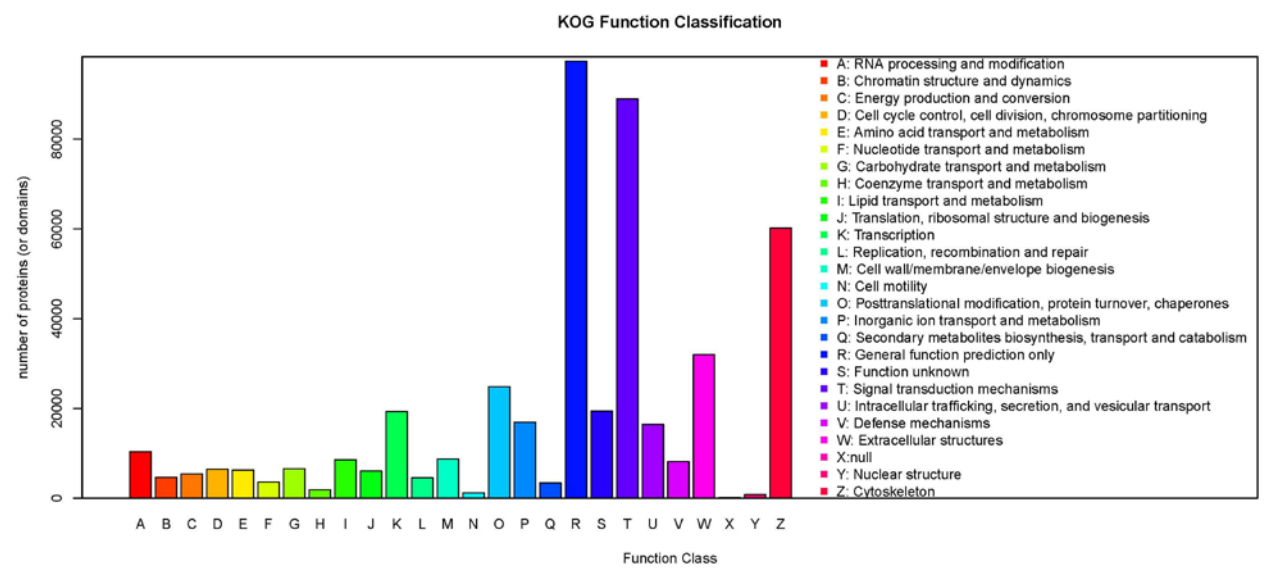

Figure 4. KOG function classification of assembled unigenes.

\section{Identification of IncRNAs}

Recent reports show that a significant proportion of noncoding RNAs could be revealed from transcriptomic data (Carninci et al., 2005; Katayama et al., 2005). IncRNAs as a key group of noncoding RNAs have gained wide attention. In the present study, we obtained 82,129 non-redundant unigenes as putative lncRNAs from sturgeon transcriptome through use of customized pipelines (Figure 6). Although lncRNA sequence tends to maintain the consensus secondary structure through compensatory base mutations, a total of $1341 \mathrm{lncRNAs}$ can still be discovered by sequence similarity comparison in A. schrenckii conserved with other vertebrates, including 391 human ortholog and 943 mouse ortholog. As expected, the 
conservation of these lncRNAs was substantially lower, only $1.63 \%$. Based on the analysis of the genomic location of conserved regions in the model organism, conserved lncRNA in sturgeon were classified into three categories: 72 lncRNAs, 283 mRNA like, and 986 other types. Detailed category information is summarized in Table 4.

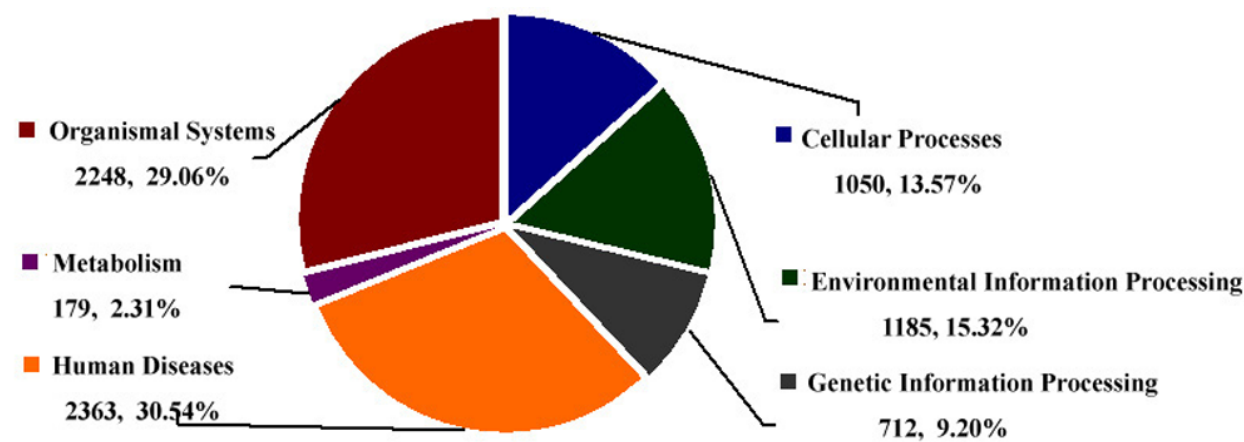

Figure 5. Classification exhibition with pie chart by KEGG pathway-enrichment analysis.

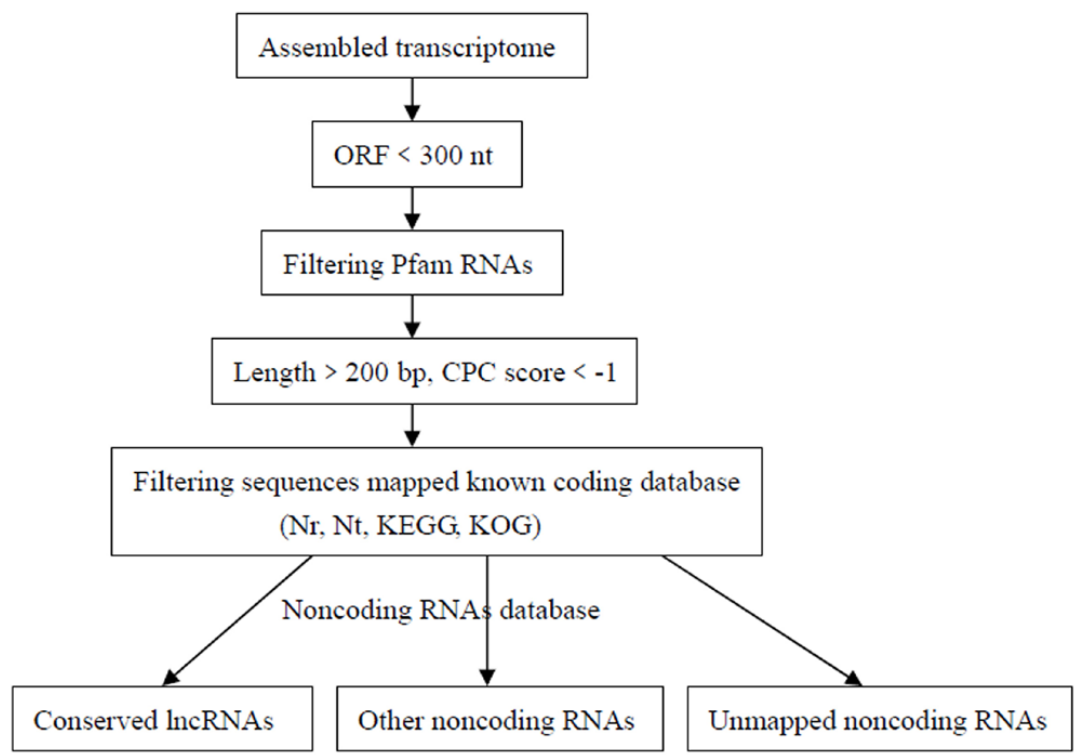

Figure 6. Customed pipelines for the identification of sturgeon lncRNAs.

Table 4. Detailed information on putative lncRNAs conservation.

\begin{tabular}{l|c|l|c|l|c|l|c}
\hline Noncoding RNA & Number (\%) & Unigene type & Number & Organism & Number & lncRNA type & Number \\
\hline Conserved lncRNAs & $1341(1.63 \%)$ & Clusters & 28 & Homo sapiens & 381 & lincRNAs & 72 \\
\hline & & & & & & mRNA like & 283 \\
\hline Others & $27(0.03 \%)$ & Singletons & 1313 & Mus musculus & 943 & Others & 986 \\
\hline
\end{tabular}




\section{DISCUSSION}

High throughput transcriptome sequencing (RNAseq) has been widely applied in numerous areas of investigation, such as alternative splice variants, gene expression profiles, noncoding RNAs, and novel transcript identification (Huang and Marth, 2008; Morozova et al., 2009). Transcriptome analysis also provides an invaluable resource for non-model species, which are scarce in genomic information, such as Chinese salamander (Che et al., 2014) and Oriental river prawn (Jin et al., 2013). In the present study, we provide the transcriptome dataset from the critically endangered Amur sturgeon (A. schrenckii). In total, 148,817 non-redundant unigene sequences with the mean length of $1599 \mathrm{bp}$ were assembled. Furthermore, all the unigenes were annotated with various known protein databases, and used to identify the SSRs and putative lncRNAs. The results should be meaningful for further investigation in many fields, including the evolution, conservation managements, and biological processes of sturgeon.

Among different sturgeon species chromosome numbers are variable and different degrees of ploidy were caused by genome duplication events (Ludwig et al., 2001). To date, genomic information of sturgeon species remains unknown, which is a negative factor for further investigation in numerous biological processes. Recently, related studies on transcriptome analysis in other sturgeon species have been conducted. For example, earlier studies on A. fulvescens (Hale et al., 2009) and A. naccarii (Vidotto et al., 2013) using Roche's 454 platform and latest studies on $A$. sinensis (Yue et al., 2015) using the Illumina platform. In the sequencing of the three sturgeon species, gonad tissues (ovary and testis) were employed for transcriptome analysis, with brain tissues included in A. naccarii. The 86,027, 55,282, and 112,504 transcripts were assembled from A. sinensis, A. naccarii, and A. fulvescens, respectively. To acquire general genetic information from $A$. schrenckii, we used five major tissue types (brain, heart, liver, spleen, and muscle) in a pooled input sample used to perform transcriptome analysis by the Illumina Hiseq 2000 platform. The number of assembled transcripts from $A$. schrenckii was markedly higher than that of the other sturgeon species.

We generated a systematic annotation for all the unigenes de novo assembled transcriptome analysis from $A$. schrenckii using three authoritative function databases (GO, $\mathrm{COG}$, and KEGG pathway). GO analysis indicated the 52 functional groups were significantly enriched (Figure 3). Comparison of enriched functional groups in A. schrenckii with those in A. fulvescens (Hale et al., 2009) revealed common GO terms, including 7 terms in molecular function, 2 in biological process and 6 in cellular component. Transcriptome data also shared similar characteristics in COG classification between A. schrenckii and A. sinensis, which showed that the unigenes involved in general function prediction only groups that were most abundant, while the unigenes participating in cell motility and nuclear structure were smaller groups. The KEGG pathway can provide a better understanding of complex networks in regulation function. In the present study, we identified 6 function categories as significant enrichment from all the 128 pathways (Figure 4). The other unmatched unigenes may represent novel genes whose functions have not yet been identified, which may play important roles in the growth and development of Amur sturgeon. However, further studies are required to elucidate these aspects. Collectively, the similarity and difference in functional annotation composition from the four sturgeon species could enrich and broaden variable genetic resources in sturgeon.

Another point of interest from transcriptome analysis is to identify noncoding RNAs. lncRNAs were first reported to have larger numbers than mammalian transcriptome, including 
human and mouse (Okazaki et al., 2002; Ota et al., 2004). Then, 1133 lncRNAs were systematically identified from zebrafish species (Pauli et al., 2012). Although they are generally lower in frequency in sequence conservation, these specific lncRNAs strongly suggest similar function conservation among different invertebrate species. For example, four evolutionary constrained mouse lncRNAs were shown to be conserved in sequence and expression between mouse, opossum, and chicken (Chodroff et al., 2010). Forty-three putative lncRNA sequences from chicken ESTs were found to share sequence homology with human, rat, and mouse transcripts (Hubbard et al., 2005). Twenty-nine detected lncRNAs in zebrafish showed high sequence and function conservation with other mammalian during embryonic development (Ulitsky et al., 2011). In this study, we also detected 1341 putatively conserved lncRNAs from $A$. schrenckii transcriptome based on sequence similarity aligned analysis, which showed that there was more than an $86.57 \%$ sequence fragment identity level with lncRNAs in the NONCODE database (Table 4). The sequence-conserved lncRNAs with other vertebrate species were only $1.63 \%$ of the total, which is much lower than that shown in related analysis (Basu et al., 2013). lncRNAs identified from sturgeon can provide a genetic resource to further investigate the mechanisms of biological processes. A total of 12,151 SSRs were identified from $A$. sinensis, in which $65.39 \%$ were a mono-nucleotide motif, which might be caused by sequencing. Therefore, only 4205 (34.61\%) SSRs with di-, tri-, tetra-, and penta-motifs would be suitable for polymorphic microsatellite locus development (Yue et al., 2015). In the present study, we acquired much larger numbers of SSRs from A. schrenckii transcriptome than those of $A$. sinensis. The di-nucleotide repeat motif was the largest group in all the types, which accounted for $53.67 \%$ of 19,860 SSRs (Table 3). The dataset will contribute to the development of microsatellite markers for A. schrenckii to aid effective conservation of this critically endangered species.

\section{Conflicts of interest}

The authors declare no conflict of interest.

\section{ACKNOWLEDGMENTS}

Research supported by the Natural Science Fund of Guangdong Province (\#2015A030313761), the Outstanding Youth Science and Technology Talent Fund of Guangdong Academy of Sciences (\#rcjj201402), and the Funds for Environment Construction \& Capacity Building of GDAS' Research Platform (\#xm2015081). We thank Mr. Wenhua Wu for helping with sturgeon sampling.

\section{REFERENCES}

Basu S, Müller F and Sanges R (2013). Examples of sequence conservation analyses capture a subset of mouse long noncoding RNAs sharing homology with fish conserved genomic elements. BMC Bioinformatics 14 (Suppl 7): S14.

Bemis WE, Findeis EK and Grande L (1997). An overview of Acipenseriformes. In Sturgeon Biodiversity and Conservation, 25-71.

Carninci P, Kasukawa T, Katayama S, Gough J, et al.; FANTOM Consortium; RIKEN Genome Exploration Research Group and Genome Science Group (Genome Network Project Core Group) (2005). The transcriptional landscape of the mammalian genome. Science 309: 1559-1563. http://dx.doi.org/10.1126/science.1112014 
Che R, Sun Y, Wang R and Xu T (2014). Transcriptomic analysis of endangered Chinese salamander: identification of immune, sex and reproduction-related genes and genetic markers. PLoS One 9: e87940. http://dx.doi.org/10.1371/ journal.pone. 0087940

Chodroff RA, Goodstadt L, Sirey TM, Oliver PL, et al. (2010). Long noncoding RNA genes: conservation of sequence and brain expression among diverse amniotes. Genome Biol. 11: R72. http://dx.doi.org/10.1186/gb-2010-11-7-r72

Conesa A, Götz S, García-Gómez JM, Terol J, et al. (2005). Blast2GO: a universal tool for annotation, visualization and analysis in functional genomics research. Bioinformatics 21: 3674-3676. http://dx.doi.org/10.1093/bioinformatics/bti610

Garg R, Patel RK, Tyagi AK and Jain M (2011). De novo assembly of chickpea transcriptome using short reads for gene discovery and marker identification. DNA Res. 18: 53-63. http://dx.doi.org/10.1093/dnares/dsq028

Grabherr MG, Haas BJ, Yassour M, Levin JZ, et al. (2011). Full-length transcriptome assembly from RNA-Seq data without a reference genome. Nat. Biotechnol. 29: 644-652. http://dx.doi.org/10.1038/nbt.1883

Hale MC, McCormick CR, Jackson JR and Dewoody JA (2009). Next-generation pyrosequencing of gonad transcriptomes in the polyploid lake sturgeon (Acipenser fulvescens): the relative merits of normalization and rarefaction in gene discovery. BMC Genomics 10: 203. http://dx.doi.org/10.1186/1471-2164-10-203

Hu J, Zhou X and Li J (2010). Development of novel EST-SSR markers for cucumber (Cucumis sativus) and their transferability to related species. Sci. Hortic. 125: 534-538. http://dx.doi.org/10.1016/j.scienta.2010.03.021

Huang W and Marth G (2008). EagleView: a genome assembly viewer for next-generation sequencing technologies. Genome Res. 18: 1538-1543. http://dx.doi.org/10.1101/gr.076067.108

Hubbard SJ, Grafham DV, Beattie KJ, Overton IM, et al. (2005). Transcriptome analysis for the chicken based on 19,626 finished cDNA sequences and 485,337 expressed sequence tags. Genome Res. 15: 174-183. http://dx.doi. org/10.1101/gr.3011405

Jin S, Fu H, Zhou Q, Sun S, et al. (2013). Transcriptome analysis of androgenic gland for discovery of novel genes from the oriental river prawn, Macrobrachium nipponense, using Illumina Hiseq 2000. PLoS One 8: e76840. http://dx.doi. org/10.1371/journal.pone.0076840

Katayama S, Tomaru Y, Kasukawa T, Waki K, et al.; RIKEN Genome Exploration Research Group; Genome Science Group (Genome Network Project Core Group); FANTOM Consortium (2005). Antisense transcription in the mammalian transcriptome. Science 309: 1564-1566. http://dx.doi.org/10.1126/science.1112009

Kong L, Zhang Y, Ye ZQ, Liu XQ, et al. (2007). CPC: assess the protein-coding potential of transcripts using sequence features and support vector machine. Nucleic Acids Res. 35: W345-9. http://dx.doi.org/10.1093/nar/gkm391

Li T, Wang S, Wu R, Zhou X, et al. (2012). Identification of long non-protein coding RNAs in chicken skeletal muscle using next generation sequencing. Genomics 99: 292-298. http://dx.doi.org/10.1016/j.ygeno.2012.02.003

Ludwig A, Belfiore NM, Pitra C, Svirsky V, et al. (2001). Genome duplication events and functional reduction of ploidy levels in sturgeon (Acipenser, Huso and Scaphirhynchus). Genetics 158: 1203-1215.

Morozova O and Marra MA (2008). Applications of next-generation sequencing technologies in functional genomics. Genomics 92: 255-264. http://dx.doi.org/10.1016/j.ygeno.2008.07.001

Morozova O, Hirst M and Marra MA (2009). Applications of new sequencing technologies for transcriptome analysis. Аnпu. Rev. Genomics Hum. Genet. 10: 135-151. http://dx.doi.org/10.1146/annurev-genom-082908-145957

Okazaki Y, Furuno M, Kasukawa T, Adachi J, et al.; FANTOM Consortium; RIKEN Genome Exploration Research Group Phase I \& II Team (2002). Analysis of the mouse transcriptome based on functional annotation of 60,770 fulllength cDNAs. Nature 420: 563-573. http://dx.doi.org/10.1038/nature01266

Ota T, Suzuki Y, Nishikawa T, Otsuki T, et al. (2004). Complete sequencing and characterization of 21,243 full-length human cDNAs. Nat. Genet. 36: 40-45. http://dx.doi.org/10.1038/ng1285

Ozsolak F and Milos PM (2011). RNA sequencing: advances, challenges and opportunities. Nat. Rev. Genet. 12: 87-98. http://dx.doi.org/10.1038/nrg2934

Pauli A, Valen E, Lin MF, Garber M, et al. (2012). Systematic identification of long noncoding RNAs expressed during zebrafish embryogenesis. Genome Res. 22: 577-591. http://dx.doi.org/10.1101/gr.133009.111

Richards VP, Suzuki H, Stanhope MJ and Shivji MS (2013). Characterization of the heart transcriptome of the white shark (Carcharodon carcharias). BMC Genomics 14: 697. http://dx.doi.org/10.1186/1471-2164-14-697

Senturk Akfirat F and Uncuoglu AA (2013). Genetic diversity of winter wheat (Triticum aestivum L.) revealed by SSR markers. Biochem. Genet. 51: 223-229. http://dx.doi.org/10.1007/s10528-012-9557-6

Tatusov RL, Fedorova ND, Jackson JD, Jacobs AR, et al. (2003). The COG database: an updated version includes eukaryotes. BMC Bioinformatics 4: 41. http://dx.doi.org/10.1186/1471-2105-4-41

Ulitsky I, Shkumatava A, Jan CH, Sive H, et al. (2011). Conserved function of lincRNAs in vertebrate embryonic development despite rapid sequence evolution. Cell 147: 1537-1550. http://dx.doi.org/10.1016/j.cell.2011.11.055

Vidotto M, Grapputo A, Boscari E, Barbisan F, et al. (2013). Transcriptome sequencing and de novo annotation of the 
critically endangered Adriatic sturgeon. BMC Genomics 14: 407. http://dx.doi.org/10.1186/1471-2164-14-407

Wei W, Qi X, Wang L, Zhang Y, et al. (2011). Characterization of the sesame (Sesamum indicum L.) global transcriptome using Illumina paired-end sequencing and development of EST-SSR markers. BMC Genomics 12: 451. http://dx.doi. org/10.1186/1471-2164-12-451

Yang SS, Tu ZJ, Cheung F, Xu WW, et al. (2011). Using RNA-Seq for gene identification, polymorphism detection and transcript profiling in two alfalfa genotypes with divergent cell wall composition in stems. BMC Genomics 12: 199. http://dx.doi.org/10.1186/1471-2164-12-199

Young RS, Marques AC, Tibbit C, Haerty W, et al. (2012). Identification and properties of 1,119 candidate lincRNA loci in the Drosophila melanogaster genome. Genome Biol. Evol. 4: 427-442. http://dx.doi.org/10.1093/gbe/evs020

Yuan L, Zhang X, Li L, Jiang H, et al. (2014). High-throughput sequencing of microRNA transcriptome and expression assay in the sturgeon, Acipenser schrenckii. PLoS One 9: e115251. http://dx.doi.org/10.1371/journal.pone.0115251

Yue H, Li C, Du H, Zhang S, et al. (2015). Sequencing and De Novo Assembly of the Gonadal Transcriptome of the Endangered Chinese Sturgeon (Acipenser sinensis). PLoS One 10: e0127332. http://dx.doi.org/10.1371/journal. pone. 0127332

Zhang J, Liang S, Duan J, Wang J, et al. (2012). De novo assembly and characterisation of the transcriptome during seed development, and generation of genic-SSR markers in peanut (Arachis hypogaea L.). BMC Genomics 13: 90. http:// dx.doi.org/10.1186/1471-2164-13-90

Zhang X, Wu W, Li L, Ma X, et al. (2013). Genetic variation and relationships of seven sturgeon species and ten interspecific hybrids. Genet. Sel. Evol. 45: 21. http://dx.doi.org/10.1186/1297-9686-45-21 